\title{
Simulation-Based Otorhinolaryngology Emergencies Boot Camp: Part 2: Special Skills Using Task Trainers
}

\author{
Sonya Malekzadeh, MD, FACS; Ellen S. Deutsch, MD, FACS; Kelly M. Malloy, MD, FACS
}

Key Words: Simulation, boot camp, resident education, cricothyrotomy, laryngoscopy, bronchoscopy, epistaxis, emergency, medical education, surgical education.

Laryngoscope, 124:1566-1569, 2014

\section{INTRODUCTION}

This second article in a series of three describes four of the eight total learning stations provided to novice otorhinolaryngology (ORL) residents during the ORL Emergencies Boot Camp. Part 1 described the overall boot camp design and the basic airway skills stations. ${ }^{1}$ This article, part 2, describes stations designed to teach moderately complex emergency skills. The final installment will address combining technical skills with decision making and teamwork, and principles for managing additional typical emergency phone calls and consults. The participants are entering ORL residents, typically but not exclusively at postgraduate year 2. ORL faculty have varying backgrounds and years of experience; the ratio of faculty to residents is approximately $1: 2 .^{2}$ Residents were surveyed about their experiences and comfort with various procedures before, immediately following, and 6 months after the boot camp. Residents found the course useful for developing knowledge, technical skills, self-confidence, and improving clinical performance for each of the skills described. ${ }^{3}$

From the Department of Otolaryngology-Head and Neck Surgery (s.m.), MedStar Georgetown University Hospital, Washington, DC; Department of Anesthesia and Critical Care (E.s.D.), The Children's Hospital of Philadelphia, Philadelphia, Pennsylvania; Department of Otolaryngology-Head and Neck Surgery (K.M.M.), University of Michigan Health System, Ann Arbor, Michigan, U.S.A.

Additional Supporting Information may be found in the online version of this article.

Editor's Note: This Manuscript was accepted for publication November 13, 2013.

The course was conducted at Simulation \& Training Environment Laboratory and MedStar Georgetown University Hospital.

The authors have no funding, financial relationships, or conflicts of interest to disclose.

Send correspondence to Kelly M. Malloy, MD, Department of Otolaryngology-Head and Neck Surgery, University of Michigan Health System, 1500 E. Medical Center Drive, First Floor, Taubman Center, Ann Arbor, MI 48109. E-mail: kellymal@umich.edu

DOI: 10.1002/lary.24571
The design of each of these skills stations is described below, with information addressing the educational premise, the handout for participants, recommended simulators, a description and checklist for additional equipment and supplies needed for the simulation, station setup, and an illustrative photograph. Handouts for each station are succinct summaries limited to one page and include learning objectives, basic information addressing the indications and the performance of the procedure, equipment needs, and clinical pearls.

\section{DIRECT (RIGID) LARYNGOSCOPY AND BRONCHOSCOPY}

Although rigid laryngoscopy and bronchoscopy are not necessarily skills that a novice otolaryngologist would use in an emergency situation, beginning to develop proficiency in this art will allow the learner to visualize anatomic details of the airway in a manner that provides important information to improve his or her understanding of airway anatomy and techniques of airway control. This station allows learners to assemble and manipulate basic rigid endoscopy instruments, as well as practice the hand-eye coordination required to integrate the tactile information received from the instruments with the visual information observed on the endoscopy monitor (see Handout, Supplementary Information 1).

As many of the manikins are stiffer and less flexible than actual patients, care must be taken to prevent damage to delicate telescopes. Although no damage to telescopes has been noted during the courses, we recommend that the telescopes be sheathed within bronchoscopes as a precaution. Similarly, care must be taken to not damage the manikins; we recommend the use of manikin lubricant and reinforce the importance of using a gentle technique. A variety of simulators are used; these can be staged in the same location as the basic airway skill station, using the same or similar manikins to minimize turnover effort (Fig. 1). 
Fig. 1. Learner performing rigid bronchoscopy on a manikin with faculty observing.

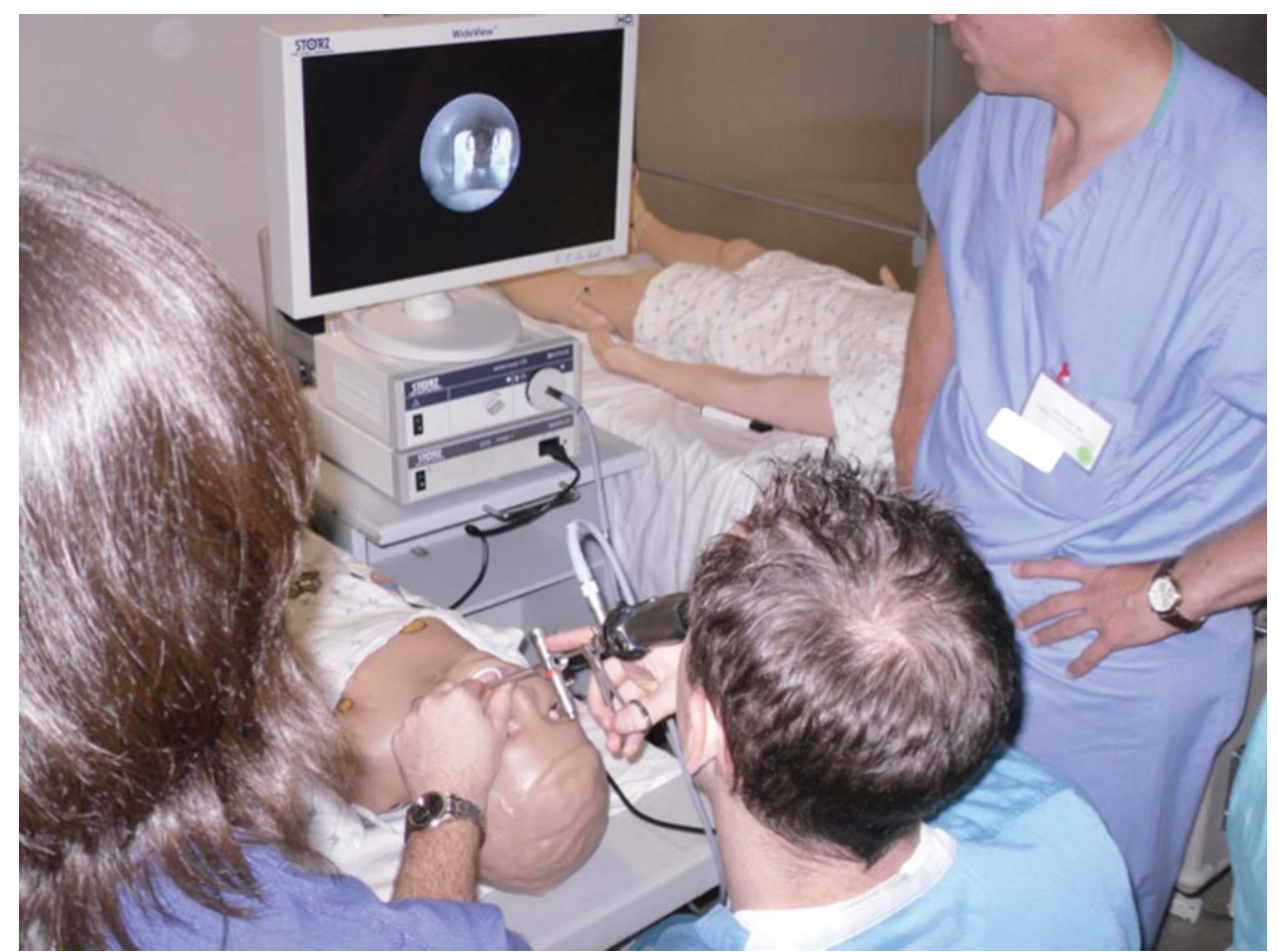

An assortment of rigid telescopes, bronchoscopes, and the relevant accessories, including bridges, prisms, and suctions, is necessary (Fig. 2). Consider including a rigid esophagoscope for comparison (Supplementary Information 2).

\section{REMOVAL OF PHARYNGEAL FOREIGN BODY}

Systematic examination of the pharynx and larynx demands an ability to coordinate the use of a headlight, tongue blade or sweet heart retractor, and/or laryngeal mirror. The additional skills of bimanual dexterity and handling of multiple instruments is required for success-

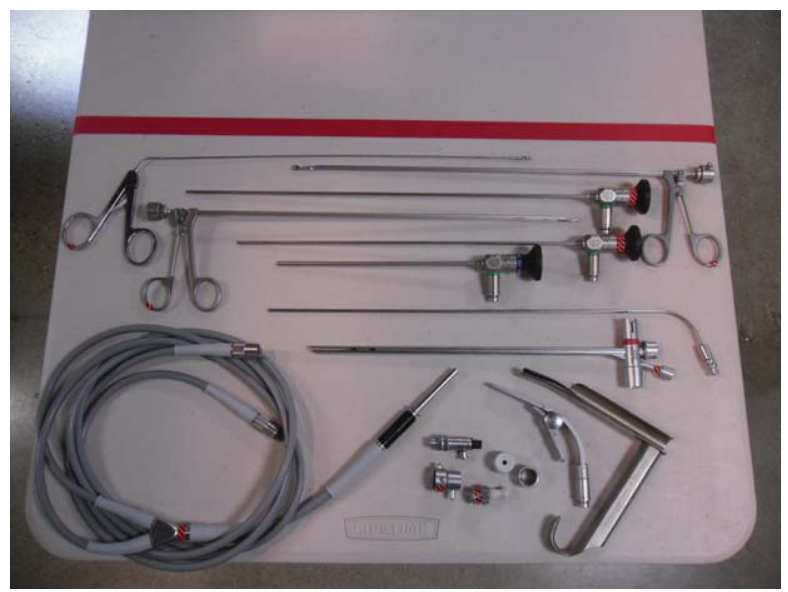

Fig. 2. Direct laryngoscopy and rigid bronchoscopy equipment setup. ful removal of a pharyngeal foreign body or other intraoral procedures such as drainage of a peritonsillar abscess (see Handout, Supplementary Information 3).

A nonelectronic adult airway trainer manikin is positioned in an upright position in either a gurney or chair raised to eye level. The faculty member places a straight pin in either the right or left inferior pole of the tonsil, mimicking a pharyngeal foreign body such as a fishbone. The participant must locate and extract the foreign body (Fig. 3). Equipment and supplies are listed in Supplementary Information 4.

\section{EPISTAXIS}

The epistaxis skills station addresses the basic skills of operating a headlight, handling a nasal speculum, and using a nasal suction device. Various nasal packing methods are briefly reviewed, and an assortment of materials is available for the exercise (see Handout, Supplementary Information 5).

The epistaxis simulator is modified from a previously described model. ${ }^{4}$ Based on our simulation center's inventory, we used the Airway Management Trainer (Laerdal, Wappingers Falls, NY), a nonelectronic adult airway trainer. We exposed the manikin's cranium and underlying facial structures by unzipping the posterior scalp skin and pulling it anteriorly. This degloving reveals the nasal dorsum. A small stab incision near the nasion with an 11 blade, followed by a spreading of the silicone tissue creates a small opening into the nasal cavity, through which the cut end of intravenous (IV) tubing is tunneled. Care should be taken to position the tube anteriorly to avoid back flow into to the nasopharynx and oropharynx; then the tubing is secured with 


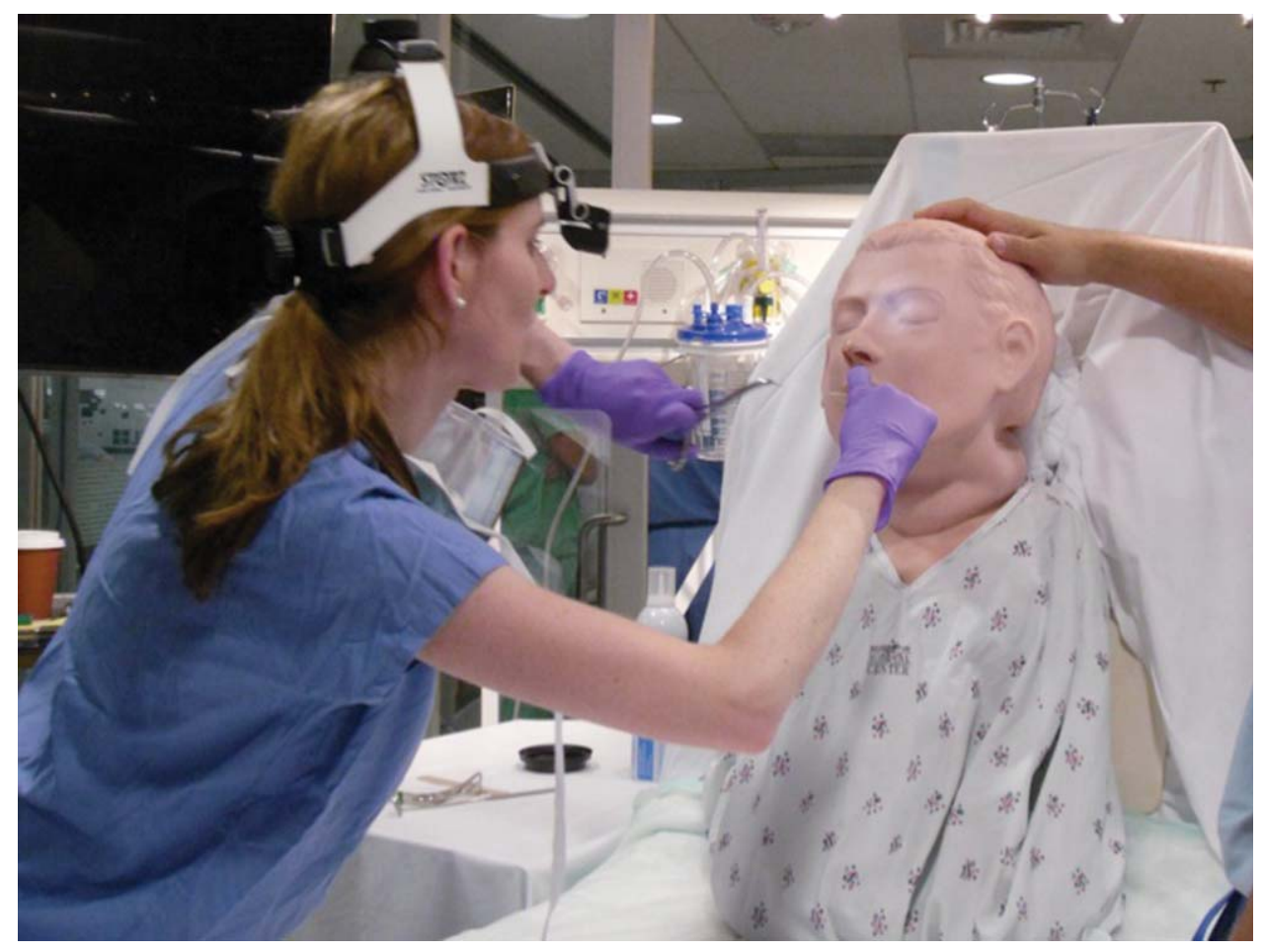

Fig. 3. Learner removing a foreign body from a manikin's pharynx. silicone adhesive. Connect the other end of the tubing to a $250 \mathrm{~mL}$ bag of fake blood that hangs from an IV pole. The blood flows by gravity and the rate is controlled with the roller clamp.

To minimize turnover effort, this can be set up in the station previously used for the pharyngeal foreign body exercise, hiding the bag of blood until needed (Fig. 4). With the faculty member controlling blood flow, the resident evaluates the nasal cavity, determines the site of bleeding, and uses various techniques to control the hemorrhage with the headlight, suction, and nasal speculum. Equipment and supplies are listed in Supplementary Information 6 .

\section{CRICOTHYROIDOTOMY AND \\ TRACHEOSTOMY TUBE CHANGE}

The cricothyroidotomy skills station familiarizes the junior residents with laryngotracheal anatomy and placement of an emergency surgical airway. Using a pig larynx model, participants perform a cricothyroidotomy, percutaneous cricothyroidotomy, tracheostomy, and tracheostomy tube change with or without the Seldinger

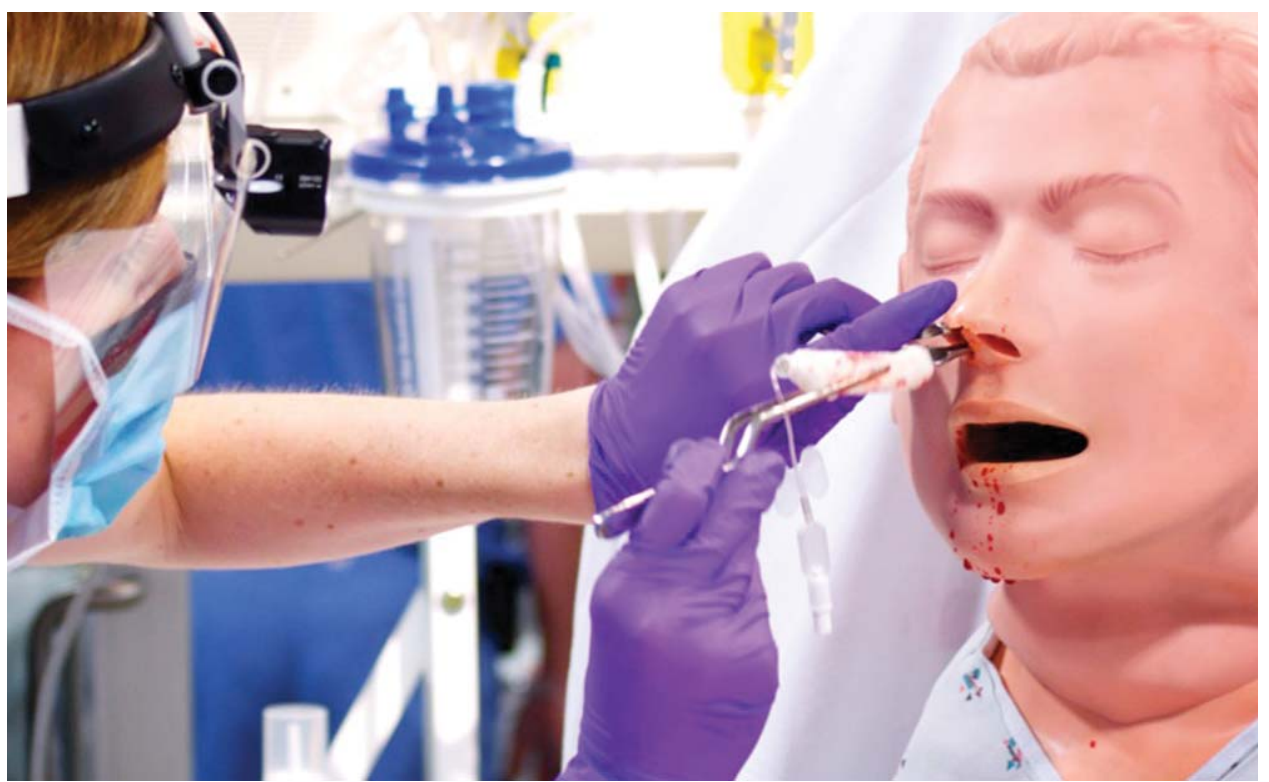

Fig. 4. Learner examining a manikin's nasal cavity and placing packing for control of epistaxis. Photo courtesy of SITEL. 


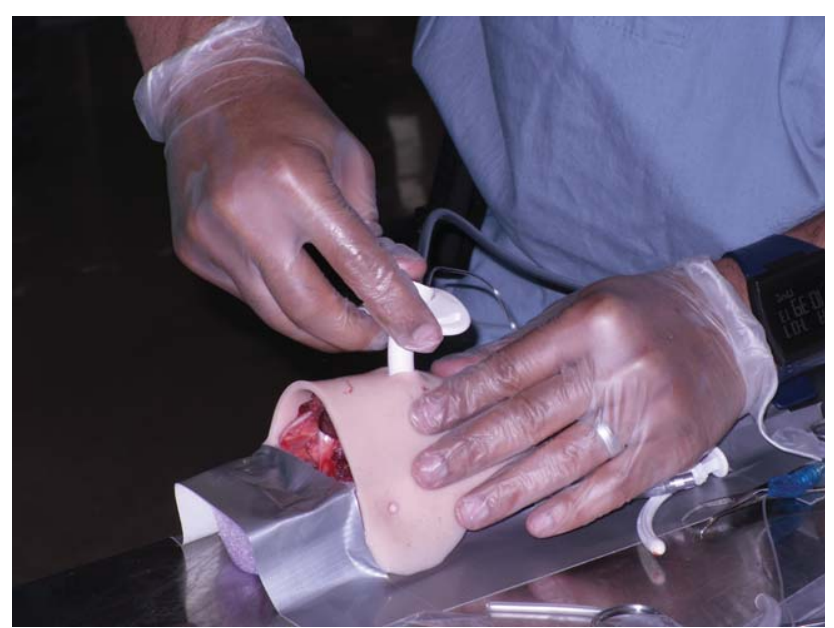

Fig. 5. Photo demonstrating a tracheostomy tube change on the pig laryngotracheal model.

method (Fig. 5) (see Handout, Supplementary Information 7 and 8).

Typically, a local or regional butcher is willing to harvest and save the specimens for a nominal fee; they may be stored frozen until needed. The pig larynx is covered with a synthetic skin fabricated from silicone (Fig. 6) or a clear nonsterile surgical glove. The assembly can be stabilized in a Styrofoam "noodle" bed. Equipment and supplies are listed in Supplementary Information 9.

In a subsequent article, "Simulation-Based Otorhinolaryngology Emergencies Boot Camp Part 3," we will address teamwork scenarios, management of additional typical emergencies and consults, and conclusions.

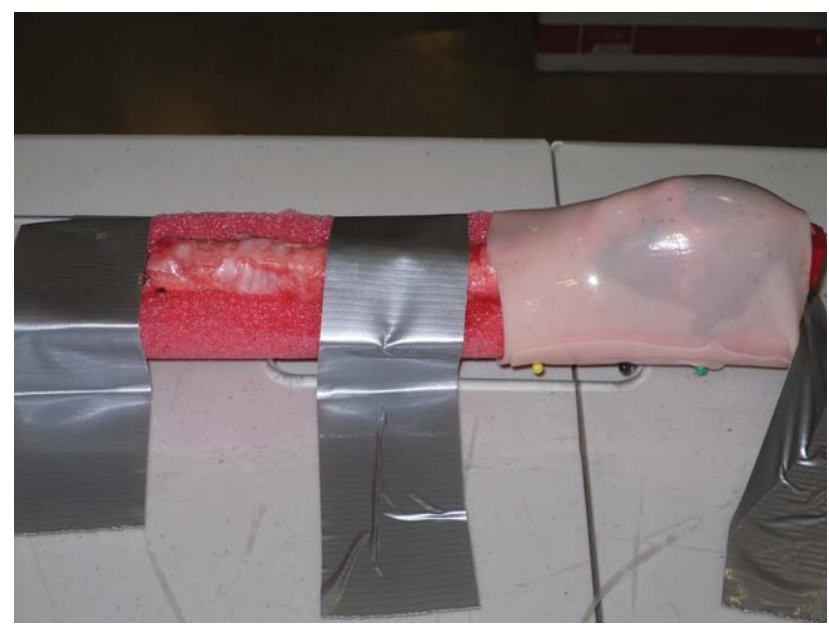

Fig. 6. Photo demonstrating the pig laryngotracheal model used for surgical airway procedures. The larynx and trachea are stabilized in a Styrofoam "swim noodle" cradle and covered with silicone skin to obscure direct visualization while maintaining palpable landmarks.

\section{BIBLIOGRAPHY}

1. Malloy KM, Malekzadeh S, Deutsch ES, Simulation-based otorhinolaryngology emergencies boot camp part 1: curriculum design and airway skills. Laryngoscope 2014;XXX:XXXX-XXXX

2. Deutsch ES, Orioles A, Kreicher K, Malloy KM, Rodgers DL. A qualitative analysis of faculty motivation to participate in otolaryngology simulation boot camps. Laryngoscope 2013:123:890-897.

3. Malekzadeh S, Malloy KM, Chu EE, Tompkins J, Battista A, Deutsch ES. ORL emergencies boot camp: using simulation to onboard residents. Laryngoscope 2011;121:2114-2121.

4. Pettineo CM, Vozenilek JA, Kharasch M, Wang E, Aitchison P. Epistaxis simulator. Simul Healthc. 2008;3:239-241. 